\title{
ACIDENTE VASCULAR ENCEFÁLICO COMO COMPLICAÇÃO DA HIPERTENSÃO ARTERIAL: QUAIS SÃO OS FATORES INTERVENIENTES?
}

\author{
Stroke as complication of arterial hypertension: which are the intervenient factors? \\ Accidente Vascular Encefálico como complicación de la hipertensión arterial: ¿cuáles \\ son los factores que interfieren?
}

\section{RESUMO}

0 objetivo foi identificar os fatores intervenientes na adesão ao tratamento anti-hipertensivo que contribuíram para surgimento do acidente vascular encefálico (AVE). Estudo descritivo realizado em uma Unidade de AVE de um hospital público. A amostra constituiuse de 75 pacientes com diagnóstico de Hipertensão Arterial Sistêmica acometidos por um AVE. A coleta de dados aconteceu em março e abril de 2010. Estudo aprovado pelo Comitê de Ética e Pesquisa sob parecer $n^{0} 110302 / 10$. Os fatores intervenientes na adesão do paciente ao tratamento anti-hipertensivo foram: sexo masculino $(61,4 \%)$, idade $>60$ anos (48\%), antecedentes familiares para HAS (68\%), baixa escolaridade (72\%), renda familiar até um salário mínimo (52\%) e presença de efeitos colaterais (22,6\%). Talvez a experiência de um AVE seja um fator determinante para a maior adesão do paciente ao tratamento antihipertensivo. É necessário que os enfermeiros realizem acompanhamento ao hiper tenso periodicamente, informando-o acerca da doença e do tratamento, visando evitar complicações.

Palavras-chave: Hipertensão. Cuidados de enfermagem. Acidente cerebral vascular.

\begin{abstract}
The objective was to identify the intervenient factors in the adhesion to the anti-hypertensive treatment that contributed to the stroke appearance as complication of arterial hypertension. This is a descriptive quantitative study carried out in a stroke unit of a public hospital. The sample was composed by 75 patients with diagnosis of systemic arterial hypertension (SAH) attacked by a stroke, admitted in the referred unit. Data collection happened in March and April 2010. The study was approved by the Research and Ethics Committee under protocol $n^{\circ} .110302 / 10$. The intervenient factors in patients' adhesion to the anti-hypertensive treatment were: male $(61.4 \%)$, older than 60 years-old $(48 \%)$, family history of SAH (68\%), low education (72\%), family income up to one minimum wage (52\%), unsatisfactory professionalpatient relationship (14.6\%), difficult access to health unit (6.6\%) and presence of side effects (22.6\%). Maybe the experience of a stroke is a decisive factor to patient's adherence to antihypertensive treatment. It is necessary that nurses carry out monitoring the patients with hypertension regularly, informing them about the disease and treatment to prevent complications.
\end{abstract}

Keywords: Hypertension. Nursing Care. Stroke.

\begin{abstract}
Resumen
El objetivo fue identificar los factores que intervienen en la adherencia al tratamiento antihipertensivo que contribuyeron a la aparición del accidente vascular encefálico (ACV). Estudio descriptivo en una unidad de ACV de un hospital público. La muestra fue constituida por 75 pacientes diagnosticados con Hipertensión Arterial Sistémica afectados por el ACV. La recolección de datos ocurrió entre marzo y abril de 2010. Estudio aprobado por el Comité de Ética e Investigación bajo parecer $n^{0} 110302 / 10$. Los factores que intervienen en la adhesión del paciente al tratamiento antihipertensivo fueron: sexo masculino $(61,4 \%)$, edad > 60 años $(48 \%)$, antecedentes familiares de hipertensión arterial (68\%), baja escolaridad (72\%), renta familiar hasta un sueldo mínimo (52\%) y presencia de efectos colaterales (22,6\%). Tal vez la experiencia de un ACV sea un factor determinante en la mayor adherencia del paciente al tratamiento antihipertensivo. Es necesario que enfermeros realicen seguimiento periódicamente, informándoles acerca de la enfermedad y del tratamiento para evitar complicaciones.
\end{abstract}

Palabras clave: Hipertensión. Atención de Enfermería. Accidente Cerebrovascular

\footnotetext{
${ }^{1}$ Enfermeira. Mestranda em Enfermagem pela Universidade Federal do Ceará (UFC). Enfermeira assistencial do Hospital Geral Dr. César Cals. Fortaleza-CE. Brasil. Email: laraenfermagem@hotmail.com; ${ }^{2}$ Enfermeira. Doutora em Enfermagem pela UFC. Professora adjunta da UFC. Fortaleza-CE. Brasil. Email: felisangela@yahoo.com.br; ${ }^{3}$ Mestre em Enfermagem. Doutoranda em Enfermagem pela UFC. Enfermeira da Secretaria de Saúde do Município. FortalezaCE. Brasil. Email: karanini@yahoo.com.br
} 


\section{INTRODUÇÃO}

A hipertensão arterial sistêmica (HAS) é considerada o maior problema de saúde pública dos países desenvolvidos e países emergentes, seja por sua alta prevalência na população adulta ou por ser considerada o principal fator de risco para o surgimento de doenças cardiovasculares'.

Em um estudo realizado no Ceará foi verificada a pressão arterial (PA) de 586.041 pessoas, constatando que $35 \%$ apresentavam taxas elevadas da PA no momento da aferição. Esse percentual é mais elevado na capital cearense, uma vez que foi verificada a pressão arterial de 137.363 , das quais $38 \%$ apresentavam pressão arterial elevada ${ }^{1}$.

A adesão ao tratamento da HAS constitui um dos maiores desafios para o profissional de saúde, pois $40 \%$ dos pacientes hipertensos não aderem satisfatoriamente ao tratamento ${ }^{2}$. As principais causas da não adesão ao tratamento da HAS são a complexidade do regime terapêutico, duração do tratamento, falha do tratamento anterior, mudanças frequentes no tratamento, influência na qualidade de vida, crenças, desconhecimento do paciente e relacionamento com a equipe de saúde ${ }^{1}$. Estudos demonstram que esses pacientes tem baixa adesão ao tratamento, contribuindo para a piora da doença e 0 aparecimento de complicações ${ }^{3,4}$.

A não adesão ao tratamento anti-hipertensivo poderá acarretar sérias complicações cardiovasculares, como doenças coronarianas, insuficiência cardíaca e acidente vascular encefálico (AVE). As doenças cardiovasculares são responsáveis por $27 \%$ dos óbitos no Brasil e uma das maiores causas de morbimortalidade no mundo ocidental. Essa elevada incidência pode ser justificada pela alta prevalência de HAS na população brasileira ${ }^{5}$.

No Brasil, o AVE merece destaque por ser a principal causa de internações, mortalidade e disfuncionalidade, superando até mesmo outras doenças cardíacas e o câncer ${ }^{6}$. Vale ressaltar que a HAS contribui como principal fator de risco para o desencadeamento do AVE. Em contrapartida, o controle adequado da PA diminui em até sete vezes o risco de um AVE².

Os profissionais de saúde, destacando-se o enfermeiro, devem prestar uma assistência na qual viabilize o autocuidado do paciente e a sua adesão ao tratamento, visto que cada um é responsável pelo seu próprio bem-estar e sua qualidade de vida?.

0 enfermeiro, como integrante da equipe de saúde, pode auxiliar o paciente na adesão ao tratamento da HAS estimulando-o, por meio de educação em saúde, a mudar os hábitos de vida e seguir o tratamento medicamentoso prescrito, sempre respeitando a individualidade de cada um $^{7}$. Desse modo, o enfermeiro deve assumir e executar seu papel de educador em saúde em todos os âmbitos de sua atuação.

As ações educativas e terapêuticas para aumentar a adesão do paciente ao tratamento anti-hipertensivo devem ser desenvolvidas não só com os pacientes, mas também com seus familiares e a comunidade. 0 enfermeiro deve atuar estabelecendo uma relação de confiança e de parceria com a família e trabalhar para informá-la acerca da doença, suas causas, consequências, e o mais importante, a forma de enfrentá-la. Pode utilizar de todas as estratégias disponíveis para orientar, educar e motivar o paciente a fim de fazer com que este adote 0 tratamento adequadamente. As estratégias incluem: reuniões individuais, palestras, simpósios, teatros, folhetos explicativos e vídeos ${ }^{8-9}$.

Diante das evidências que indicam a baixa adesão ao tratamento anti-hipertensivo como um dos aspectos relacionados ao aparecimento de complicações devido à manutenção de valores pressóricos elevados ao longo do tempo, surgiram os seguintes questionamentos: quais os fatores intervenientes na adesão ao tratamento anti-hipertensivo e quais os contribuintes para o surgimento do AVE como complicação da hipertensão arterial?

0 presente estudo torna-se relevante, pois o impacto das doenças cardiovasculares e suas complicações, destacando a HAS e o AVE, respectivamente, sobre a sociedade é crescente, especialmente quando evolui para graus variados de incapacidade ou morte. Além disso, a identificação e o conhecimento desses fatores podem contribuir para a elaboração de um plano de cuidados de enfermagem adequado e individualizado que focalize as necessidades do paciente com o intuito de melhorar sua adesão ao tratamento da HAS e evitar complicações como o AVE.

Assim, o estudo teve como objetivo identificar os fatores intervenientes na adesão ao tratamento antihipertensivo que contribuíram para o surgimento do AVE como complicação da HAS

\section{METODOLOGIA}

Trata-se de um estudo descritivo, de natureza quantitativa, realizado na unidade de AVE de um hospital público terciário de Fortaleza-Ceará. A unidade de AVE possui 20 leitos e nela atua uma equipe multidisciplinar.

A população do estudo foi constituída por 166 pacientes com diagnóstico de HAS acometidos por AVE, internados na referida unidade no período da coleta de dados. A amostra foi definida a partir da fórmula indicada para o cálculo em estudos transversais de população finita. Foi considerado um nível de significância de $95 \%(1,96)$; a prevalência de AVE na unidade é de $73 \%$; e o erro amostral considerado foi de $5 \%$.

Considerando uma taxa de $10 \%$ de perdas, incluindo as desistências, dados incompletos e informações erradas, 0 tamanho amostral definido foi de 75 pacientes, os quais atenderam aos critérios de inclusão: ter sido acometido por um AVE; ter diagnóstico de HAS definido antes do AVE; estar internado na unidade de AVE durante o período do estudo; ser maior de 18 anos, estar em condições físicas e emocionais de participar da entrevista, respondendo aos questionamentos. 
A coleta de dados aconteceu nos meses de março e abril de 2010 por meio de uma entrevista estruturada com o paciente. Os dados foram armazenados em um banco de dados do Excel, sendo apresentados em tabelas e analisados com estatística descritiva e fundamentados na literatura pertinente à temática.

$\mathrm{Na}$ realização do estudo, foram seguidos os princípios bioéticos previstos na Resolução 196/96 do Conselho Nacional de Saúde, referentes à pesquisa envolvendo seres humanos e mediante a aprovação do Comitê de Ética da instituição investigada. Para tanto, o projeto foi aprovado pelo Comitê de Ética e Pesquisa da referida instituição, o qual emitiu parecer favorável para seu desenvolvimento, sob protocolo $n^{0} 110302 / 10$.

\section{RESULTADOS}

Durante o período da coleta de dados, foram identificados 75 pacientes que atendiam aos critérios do estudo. Os dados foram organizados em tabelas de acordo com a caracterização dos pacientes: presença de fatores não modificáveis e presença de fatores modificáveis que podem interferir na adesão ao tratamento da HAS.

Tabela 1 - Caracterização dos pacientes quanto aos fatores não modificáveis intervenientes na adesão ao tratamento da HAS antes de vivenciar um acidente vascular encefálico. Fortaleza-CE, mar-abr/2010. $N=75$.

\begin{tabular}{|c|c|c|c|}
\hline & $\mathrm{N}$ & $\%$ & Média \\
\hline \multicolumn{4}{|l|}{ Sexo } \\
\hline Masculino & 46 & 61,4 & \\
\hline Feminino & 29 & 38,6 & \\
\hline \multicolumn{4}{|l|}{ Faixa etária } \\
\hline 31 a 40 anos & 3 & 4,0 & \\
\hline 41 a 50 anos & 16 & 21,3 & \\
\hline 51 a 60 anos & 20 & 26,7 & \\
\hline$>60$ anos & 36 & 48,0 & 60 anos \\
\hline \multicolumn{4}{|c|}{ Antecedentes familiares } \\
\hline Presentes & 51 & 68,0 & \\
\hline Ausentes & 24 & 32,0 & \\
\hline \multicolumn{4}{|c|}{ Tempo de diagnóstico da HAS } \\
\hline 1 a 3 anos & 26 & 34,7 & \\
\hline 3 a 10 anos & 27 & 36,0 & \\
\hline$>10$ anos & 22 & 29,3 & \\
\hline \multicolumn{4}{|c|}{ Tempo de tratamento da HAS } \\
\hline 1 a 3 anos & 30 & 40,0 & \\
\hline 3 a 10 anos & 24 & 32,0 & \\
\hline$>10$ anos & 21 & 28,0 & \\
\hline \multicolumn{4}{|c|}{ Presença de Morbidades } \\
\hline Sim & 28 & 37,3 & \\
\hline Não & 47 & 62,7 & \\
\hline
\end{tabular}

Com base na Tabela 1, constata-se que 61,4\% dos pacientes são do sexo masculino; a faixa etária variou entre 31 e 82 anos de idade, sendo que a maioria (48\%) dos entrevistados tinha mais de 60 anos e a idade média entre eles foi de 60 anos. Com relação aos antecedentes familiares de HAS, $64 \%$ dos pacientes relataram história familiar.

Dos 75 entrevistados, $34,7 \%$ descobriram serem portadores de HAS há menos de três anos, $36 \%$ descobriram entre 3 e 10 anos e 29,3\% dos entrevistados foram diagnosticados há mais de 10 anos. Contudo, 40\% desses pacientes iniciaram o tratamento há menos de três anos, ou seja, alguns pacientes tiveram o diagnóstico de HAS, mas só iniciaram o tratamento anos depois.

As morbidades estavam presentes em $37,3 \%$ dos pacientes, dentre elas destacam-se o diabetes mellitus, as cardiopatias e as nefropatias. 
Tabela 2 - Caracterização dos pacientes quanto aos fatores modificáveis intervenientes na adesão ao tratamento da HAS antes de vivenciar um acidente vascular encefálico. Fortaleza-CE, mar-abr/ 2010. $N=75$

\begin{tabular}{lll}
\hline & $\mathrm{N}$ & $\%$ \\
\hline Escolaridade & & \\
Analfabeto & 22 & 29,3 \\
Ensino fundamental completo/incompleto & 32 & 42,7 \\
Ensino médio completo/incompleto & 21 & 28,0 \\
Estado civil & & \\
Solteiro & 18 & 24,0 \\
Casado/União estável & 57 & 76,0 \\
Renda familiar (salário mínimo) & & \\
& & \\
<1 & 39 & 52,0 \\
1 a 3 & 26 & 34,7 \\
4 a 6 & 4 & 5,3 \\
\hline > & 6 & 8,0 \\
Relação profissional-paciente no centro de saúde & & \\
Satisfatória & 64 & 85,4 \\
Insatisfatória & 11 & 14,6 \\
Acesso à unidade de saúde & & \\
Fácil & 70 & 93,4 \\
Difícil & 5 & 6,6 \\
Disponibilidade dos medicamentos nos centros de saúde & & \\
Sempre tem & & \\
As vezes falta & 46 & 61,4 \\
Quantidade de medicamentos anti-hipertensivos diários & 29 & 38,6 \\
Um & & \\
Dois & 54 & 72,0 \\
Três & 19 & 25,4 \\
Efeitos colaterais & 2 & 2,6 \\
Sim & & \\
Não & 58 & 22,6 \\
\hline & & 77,4 \\
\hline
\end{tabular}

$\mathrm{Na}$ Tabela 2 estão expostas as variáveis relacionadas aos fatores que interferem na adesão ao tratamento da HAS. Em relação ao nível escolar, 70,7\% dos entrevistados eram alfabetizados e tinham pelo menos cursado o ensino fundamental. 0 estado civil solteiro predominou em $24 \%$ dos pacientes. Em relação à renda familiar, $52 \%$ dos entrevistados recebiam até um salário mínimo, e apenas $8 \%$ recebiam mais de seis salários mínimos. 0 alto índice de pacientes com renda familiar de até um salário mínimo pode está relacionado ao alto índice de pacientes aposentados. Quanto à relação do profissional com o paciente, $14,6 \%$ consideravam a relação insatisfatória.

Quanto ao acesso dos pacientes ao centro de saúde para acompanhamento no programa de hipertensão arterial, 6,6\% afirmaram ter dificuldade de comparecer às consultas, pois o centro de saúde é distante de suas residências, sendo esse um dos motivos para a irregularidade das consultas agendadas.

No que diz respeito à disponibilidade de medicações anti-hipertensivas no centro de saúde, 38,6\% afirmaram que as medicações às vezes faltavam nas unidades de saúde.

Em relação à quantidade de medicações orais administradas diariamente, $28 \%$ dos entrevistados tomavam dois ou mais tipos de medicação. Destaca-se que, quanto mais medicamentos forem necessários para o controle da PA, mais dificuldades os pacientes apresentam em aderir ao tratamento medicamentoso.

Dos entrevistados, três faziam uso de um betabloqueador associado a um inibidor da enzima conversora de angiotensina (IECA); nove pacientes estavam recebendo um diurético associado a um IECA; três pacientes faziam uso de um diurético associado a um antagonista dos receptores de angiotensina (ARA); dois pacientes estavam em uso de dois 
tipos de diuréticos associados a um IECA; um paciente fazia uso de um IECA associado a um bloqueador dos canais de cálcio; um paciente estava recebendo um IECA associado a um ARA; um paciente fazia uso de um diurético associado a um betabloqueador; e um paciente, de um betabloqueador associado a um bloqueador dos canais de cálcio.

No que diz respeito aos efeitos colaterais, $22,6 \%$ dos pacientes apresentavam algum tipo de efeito colateral após tomar a medicação, o qual poderia interferir na continuidade do uso dos medicamentos regularmente.

\section{DISCUSSÃO}

Em relação ao sexo, 61,4\% dos entrevistados eram do sexo masculino. Com relação à adesão ao tratamento da HAS, as mulheres tendem a aderir ao tratamento melhor do que os homens provavelmente por terem um maior conhecimento sobre a doença e suas complicações e por estarem menos envolvidas com o tabagismo e o alcoolismo ${ }^{10}$.

Quanto à idade, $48 \%$ dos entrevistados tinham mais de 60 anos. Os indivíduos de idade mais avançada são mais propensos à adesão, enquanto os jovens são menos propensos visto que não se sentem vulneráveis à doença, enquanto os idosos, mais preocupados com a saúde, se apegam ao tratamento como alternativa de prolongamento da vida. A procura dos jovens pelo serviço de saúde é pouco significativa para eles, pois não se preocupam em prevenir doenças e buscam o tratamento apenas para os sintomas que incomodam ${ }^{11}$.

$\mathrm{Na}$ nossa entrevista, $32 \%$ dos pacientes não relataram história familiar de HAS. Experiências com a doença é apontada como fator preditor para a adesão ao tratamento anti-hipertensivo. A presença de um membro da família portador de HAS influi na adesão ao tratamento por parte do paciente. Isso demonstra que a ausência de um parente portador de HAS influi negativamente na adesão ao tratamento pelo paciente ${ }^{12}$.

0 tempo de diagnóstico da HAS foi muito variado, 65,3\% receberam o diagnóstico médico há mais de três anos; contudo, alguns desses pacientes só iniciaram o tratamento posteriormente. No entanto, o início do tratamento medicamentoso deve acontecer assim que for detectada a $\mathrm{HAS}^{13}$. 0 tempo de diagnóstico e tratamento influi na adesão do hipertenso ao tratamento, pois, quanto menor esse tempo, maior é a adesão do paciente ao tratamento 1 .

A morbidade mais presente entre os pacientes foi 0 diabetes mellitus $(81,4 \%)$, seguido das cardiopatias $(29,6 \%)$ e nefropatias (7,4\%). A adesão ao tratamento da HAS é menos prevalente em pacientes com morbidades associadas, como 0 diabetes mellitus ou a doença coronariana ${ }^{14}$.

No presente estudo, 0 analfabetismo estava presente em $29,3 \%$ dos pacientes, e $70,7 \%$ tinham pelo menos cursado o ensino fundamental. Observou-se em um estudo realizado com 93 pacientes portadores de HAS que a maioria dos entrevistados era analfabeta ${ }^{5}$.
0 nível de escolaridade influi diretamente na assimilação das orientações acerca da patologia, portanto, quanto mais baixa a escolaridade, mais difícil se torna compreender o diagnóstico, a necessidade da mudança de hábitos e os esquemas posológicos. Indivíduos com baixo nível de escolaridade podem ter dificuldades em compreender as orientações fornecidas pelos profissionais de saúde e, consequentemente, não aderir satisfatoriamente ao tratamento ${ }^{11}$.

Em relação ao estado civil, $24 \%$ dos pacientes eram solteiros, ressaltando que quase todos os pacientes solteiros eram do sexo feminino. Hipertensos casados, quando comparados com os solteiros, apresentam chance duas vezes maior de aderir ao tratamento proposto ${ }^{12}$.

Participando ativamente do cuidado, os cuidadores familiares desempenham papel significativo na adesão do paciente ao tratamento da HAS. A participação da família é altamente relevante na aquisição de hábitos e mudanças no estilo de vida, assim como no seguimento do tratamento farmacológico, demonstrando que é de extrema importância estar em contato com a família na busca pela adesão da pessoa ao tratamento. Ė importante também que o enfermeiro, como educador familiar, oriente os familiares do hipertenso quanto ao seguimento correto do tratamento, para que estes ajudem o paciente a desempenhar seus cuidados adequadamente ${ }^{15}$.

Quanto à renda familiar, 52\% tinham como renda familiar até um salário mínimo, dados semelhantes foram encontrados em um estudo, no qual $50 \%$ dos entrevistados eram considerados de baixa renda ${ }^{5}$. A condiçãa socioeconômica é um fator preponderante para a não adesão ao tratamento, $\mathrm{e}$ um nível socioeconômico baixo está relacionado à maior prevalência de HAS. Quanto maior o poder aquisitivo, maior é o acesso aos medicamentos; portanto, pacientes com renda mensal baixa tem mais probabilidade de não aderir ao tratamento da hipertensão $0^{14}$.

Quanto à relação do profissional com o paciente, $14,6 \%$ dos entrevistados consideravam a relação insatisfatória. Esta foi caracterizada pelo não esclarecimento de todas as dúvidas relacionadas à doença e a medicamentos e pela falta de atenção do profissional com o paciente.

A falta de uma comunicação adequada entre o paciente e o profissional sobre a doença e as sérias consequências do abandono ao tratamento podem influenciar negativamente na adesão ao tratamento. De uma forma ou de outra, fica claro que estratégias devem ser desenvolvidas para melhorar a comunicação entre paciente e profissional, com intuito de aumentar a aderência do paciente ao tratamento proposto ${ }^{14}$.

A unidade de saúde localizava-se perto da residência da maioria dos entrevistados (93,4\%). A não proximidade da unidade de saúde da residência do paciente interfere na sua adesão ao tratamento, pois ele acaba tendo mais dificuldade de comparecer às consultas agendadas e menos acesso aos medicamentos $^{12}$. 
Quanto à disponibilidade das medicações pela unidade de saúde, 38,6\% relataram que a medicação às vezes faltava na unidade. É rotineira no acompanhamento à clientela a não aderência ao tratamento medicamentoso da HAS; isto ocorre devido à falta de recursos financeiros para adquirir 0 medicamento, quando esse está em falta na instituição. Apesar de a despesa com medicamentos representar grande parte do investimento em saúde pública, em países como o Brasil, a dispensação gratuita de medicamentos não cobre a demanda'.

Com relação à quantidade de medicações administrados diariamente, $28 \%$ dos entrevistados tomavam dois ou mais tipos de medicação. A quantidade de medicamentos utilizados pode interferir na adesão do paciente ao tratamento, pois, quanto maior o número de medicações utilizados por dia, maior a probabilidade de o paciente não aderir ao tratamento medicamentoso ${ }^{10}$.

A presença de efeitos colaterais relacionadas ao uso do medicamento teve prevalência de 22,6\%. A identificação de efeitos colaterais ao tratamento farmacológico instituído representa uma causa de abandono ao tratamento ${ }^{1,14}$.

No nosso estudo os principais efeitos colaterais mencionados foram: dor no estômago, mal-estar, náuseas e tosse seca. Os sintomas descritos como efeitos colaterais pelos pacientes hipertensos em tratamento farmacológico ocorrem principalmente no início da terapia e diminuem com o tempo. Hoje existem no mercado medicações modernas e com baixo perfil de efeitos adversos, cabe ao profissional médico saber da existência desses medicamentos para que possa substituir o medicamento anterior e diminuir o abandono do tratamento medicamentoso ${ }^{16}$.

A baixa adesão ao tratamento está relacionada a fatores como problemas socioeconômicos, distúrbios psicológicos e prejuízos cognitivos somados às características da doença e complexidade do tratamento, além do custo, quantidade e efeitos das medicações e do relacionamento entre profissional e paciente ${ }^{17} .0$ fato de não se considerar doente tendo hiper tensão e não conceber essa condição como doença pode levar o indivíduo a não realizar os cuidados e não entendê-los como necessários para si, o que pode repercutir no tratamento da condição crônica $^{18}$.

Aderir ao tratamento medicamentoso da HAS também não é fácil, pois é um processo complexo que inclui a administração correta da medicação, no horário certo e na quantidade certa ${ }^{18}$.

\section{CONCLUSÕES}

Esses dados se referem à adesão do paciente com HAS antes de vivenciar um acidente vascular encefálico. Contudo, talvez a experiência de um acidente vascular encefálico seja um fator determinante para a maior adesão ao tratamento anti-hiper tensivo por parte do paciente. É importante ressaltar que a prevenção dos fatores de risco para o desenvolvimento de um acidente vascular encefálico é o primeiro passo para diminuir sua incidência e prevalência.

Buscar melhorar a adesão do indivíduo ao tratamento é, portanto, uma meta primordial no direcionamento das ações de enfermagem ao paciente com hipertensão em todos os níveis de atenção à saúde.

Os fatores intervenientes não modificáveis na adesão do paciente ao tratamento anti-hipertensivo foram: sexo masculino $(61,4 \%)$, idade maior que 60 anos (48\%), antecedentes familiares de HAS (32\%) e morbidades presentes $(37,3 \%)$.

Os fatores modificáveis foram: baixa escolaridade $(72 \%)$, estado civil solteiro (24\%), renda familiar até um salário mínimo (52\%), relação profissional-paciente insatisfatória (14,6\%), falta dos medicamentos no centro de saúde (38,6\%), difícil acesso ao centro de saúde $(6,6 \%)$, uso de mais de um medicamento anti-hipertensivo (28\%) e presença de efeitos colaterais $(22,6 \%)$. Vale destacar que a identificação de efeitos colaterais ao uso de medicamento pode contribuir como causa de abandono do tratamento.

É necessário que os enfermeiros realizem acompanhamento ao paciente com hipertensão periodicamente, informando-o acerca da doença e do tratamento por toda a vida por meio de educação em saúde, visando evitar complicações. Tal acompanhamento é necessário, visto que a adesão ao tratamento da hipertensão é um processo complexo e deve ser constante por necessitar do envolvimento e participação ativa dos pacientes para a prática do autocuidado, visando à prevenção de doenças e à promoção da saúde.

\section{REFERÊNCIAS}

1.Santos ZMSA, Frota MA, Cruz DM, Holanda SDO. Adesão do cliente hipertenso ao tratamento: análise com abordagem interdisciplinar. Texto\&Contexto Enferm. 2005; 14(3): 332-40.

2.Ferreira Filho C, Meneghini A, Riera ARP, Serpa Neto A, Teixeira GK, Gomes SR. Assistência de enfermagem à pessoa com acidente vascular cerebral. Cogitare Enferm. 2008; 13(2): 220-6.

3.Kripalani S, Yao X, Haynes B. Interventions to enhance medication adherence in chronic medical condictions. BioMed Central Cardiovascular Disorders. 2006; 6:1-8.

4.Renner SBA, Franco RR, Berlezi EM, Bertholo LC. Associação da hipertensão arterial com fatores de riscos cardiovasculares em hipertensos de ljuí, RS. Rev Bras Anal Clin 2008; 40(4): 261-66.

5.Gomes SR, Senna, M. Assistência de enfermagem a pessoa com acidente vascular cerebral. Cogitare Enferm. 2008; 13(2): 220-26. 
6.Vitor RS, Sakai FK, Consoni PRC. Indicação e adesão de medidas não farmacológicas no tratamento da hipertensão arterial. Rev AMRIGS 2009; $53(2): 117-21$.

7.Gusmao JL. Adesão ao tratamento em hipertensão arterial sistólica isolada. Rev Bras Hipertens. 2009; 16(1) :38-43.

8.Sociedade Brasileira de Hipertensão-SBH. VI Diretrizes Brasileiras de Hipertensão Arterial. São Paulo; 2010.

9.Araújo IL, Paz, EPA, Moreira, TMM. Hermenêutica e o cuidado de saúde na hipertensão arterial realizado por enfermeiros na estratégia saúde da família. Esc Anna Nery. 2010 jul/set; 14 (3): 560-66. [ citado 2010 maio 19]. Disponível em http://www.eean.ufri.br/revista enf/20103/ artigo\%560.pdf.

10.Veras RFS, Oliveira JS. Aspectos sócio-demográficos que influenciam na adesão ao tratamento anti-hipertensivo. Rev RENE. 2009; 10(3):13238.

11.Araújo GBS, Garcia TR. Adesão ao tratamento anti-hipertensivo: uma análise conceitual. Rev Eletr Enferm. 2006; 8(2): 259-72.

12.Santos Neto LL, Toledo MA, Medeiros OS. Hipertensão arterial e acidente vascular cerebra. Brasília Med. 2005; 42(1): 24-30.

13.Borges CP, Arantes RL. Aspectos epidemiológicos da aderência ao tratamento de hipertensão arterial sistêmica. Rev Med Ana Costa. 2006;11(1).

14.Saraiva KRO, Santos ZMSA, Landim FLP, Lima HP, Sena VL. 0 processo de viver do familiar cuidador na adesão do usuário hipertenso ao tratamento. Texto\& Contexto Enferm. 2007;16(1): 63-70.

15.Osterberg L, Blaschke T. Adherence to medication. New England J Med. 2005; 453: 487-97.

16.Mantovani MF, Mottin JV, Ulbrich EM, Pinotti S. Caracterização dos usuários e o conhecimento sobre a hipertensão arterial. Online Braz J Nurs. 2008 [cited 2010 Jul 07]; 7(2). Avaiable: http://www.ob jnursing .uff.br/index.php/nursing/article/view/j.1676-4285.2008.1467/348

17.Lopes MCL, Marcon SS. Concepções sobre saúde e doença de famílias que convivem com a hipertensão arterial: um estudo qualitativo. Online Braz J Nurs. 2009 [citado 2010 jul 07]; 8(3). Disponível em: http:// www.objnursing.uff.br/index.php/nursing/article/view/ j.16764285.2009.2484/html 35

18.Reiners AAO, Nogueira MS. Conscientização do usuário hipertenso para a adesão ao tratamento. Rev Latino-am Enfermagem. 2009; 17(1): 59-65. 